\title{
Long non-coding RNA AOC4P suppresses epithelial ovarian cancer metastasis by regulating epithelial-mesenchymal transition
}

Xiaojing Lin ${ }^{1,2,3}$, Xiaoyan Tang ${ }^{1,3}$, Tingting Zheng ${ }^{1,3}$, Junjun Qiu ${ }^{1,3^{*}}$ and Keqin Hua ${ }^{1,3^{*}}$

\begin{abstract}
Objective: Currently, the function and mechanisms of long non-coding RNAs (IncRNAs) involved in the metastasis of epithelial ovarian cancer (EOC), especially those of the IncRNAs participated in the epithelial-mesenchymal transition (EMT) process, remains largely unknown. Here, we focused on a IncRNA named AOC4P and analysed its role in EOC.

Materials and methods: The expression of AOC4P gene was examined with quantitative real-time quantitative PCR (qRT-PCR). The cell migration and invasion were detected by Transwell and scratch assays. The in vivo metastatic activity was evaluated by intraperitoneal metastasis model. The downstream genes were investigated by a tumour EMT real-time polymerase chain reaction (RT-PCR) array, and validated by qRT-PCR and Western blot.

Results: The results showed that AOC4P expression levels were decreased in EOC tissues and cell lines, and that the under-expression of AOC4P was positively correlated with FIGO stage and lymph node metastasis. Furthermore, the knockdown of AOC4P expression in poorly metastatic EOC cell lines remarkably facilitated cell migration/invasion while the overexpression of AOC4P in highly metastatic EOC cell lines reduced the metastatic ability of these cells in vitro. Consistently, the anti-metastatic role of AOC4P in vivo was also verified by bioluminescence imaging and tumour dissection. Mechanistically, the anti-metastatic effect of AOC4P in EOC was partially mediated by the EMT process accompanied by the alterations in MMP9 and COL1A2 expression.

Conclusion: These data highlight that AOC4P plays a critical role in EOC invasion/metastasis and could function as a novel and effective target for the IncRNA-based anti-metastatic clinical management of EOC.
\end{abstract}

Keywords: IncRNA, AOC4P, EMT, Metastasis, Ovarian cancer

\section{Introduction}

Epithelial ovarian cancer (EOC), which represents the majority of all ovarian cancers (OC), has the highest mortality rate among gynecological cancers [1]. Poor patient prognosis is primarily attributed to the widespread metastatic events [2]. Due to easy invasion of adjacent

\footnotetext{
* Correspondence: qiujunjun1113@163.com; huakeqin@fudan.edu.cn 'Department of Gynecology, Obstetrics and Gynecology Hospital of Fudan University, 419 Fangxie Road, Shanghai 200011, P.R. China

Full list of author information is available at the end of the article
}

organs or metastasis to the peritoneal cavity, the 5-year survival rate of $\mathrm{OC}$ patients remains the unsatisfactory rate of 30\% [3]. Therefore, methods for elucidating the mechanisms underlying metastasis are urgently needed.

Epithelial-mesenchymal transition (EMT) exerts a major function in initiating metastasis [4]. Evidence indicates that at the initial stage of EOC metastasis, cells undergo EMT to acquire highly invasive phenotypes, and this acquisition is accompanied by multiple molecular events involving signalling pathways, epithelial and mesenchymal

(C) The Author(s). 2020 Open Access This article is licensed under a Creative Commons Attribution 4.0 International License, which permits use, sharing, adaptation, distribution and reproduction in any medium or format, as long as you give appropriate credit to the original author(s) and the source, provide a link to the Creative Commons licence, and indicate if changes were made. The images or other third party material in this article are included in the article's Creative Commons licence, unless indicated otherwise in a credit line to the material. If material is not included in the article's Creative Commons licence and your intended use is not permitted by statutory regulation or exceeds the permitted use, you will need to obtain permission directly from the copyright holder. To view a copy of this licence, visit http://creativecommons.org/licenses/by/4.0/. The Creative Commons Public Domain Dedication waiver (http://creativecommons.org/publicdomain/zero/1.0/) applies to the data made available in this article, unless otherwise stated in a credit line to the data. 
markers (EMT markers), EMT-transcription factors (EMT-TFs), and EMT-associated inducers such as matrix metalloproteinases (MMPs) [2, 5-8].

Apart from these well-known regulators, long noncoding RNAs (lncRNAs), a primary group of non-coding RNAs, are emerging as pivotal players in EMT-associated metastasis in malignancies [9]. The copper containing 4, pseudogene (AOC4P) is a recently discovered EMTrelated IncRNA that was initially reported in hepatocellular carcinoma (HCC) [10]. Evidence has also shown the involvement of AOC4P in colorectal cancer (CRC) and gastric cancer (GC) $[11,12]$. However, studies regarding the clinical value and biological role of AOC4P in EOC have not been elucidated.

In this study, we measured the expression of AOC4P in EOC clinical samples and cell lines, and analyzed the correlations between AOC4P expression and EOC clinicopathological features. Subsequently, to probe the metastatic function of AOC4P in vitro, three pairs of poorly/highly metastatic cell lines were chosen for loss/ gain of function assays. Additionally, a nude mouse model of abdominal metastasis was established to determine the in vivo activity of AOC4P. Moreover, the molecular mechanisms underlying the AOC4P-mediated anti-metastatic effect on EOC were investigated, and experiments ascertained whether certain EMT-related factors were partially implicated in this phenotypic transformation associated with EMT. These data indicate the instructive value of AOC4P in EOC progress.

\section{Materials and methods}

\section{Patients and samples}

The study cohort consisted of 70 EOC patients and 10 patients with uterine fibroids who underwent hysterectomy and oophorectomy at the Obstetrics and Gynecology Hospital of Fudan University between August 2013 and September 2016. These 80 tissue samples (consisting of 70 EOC and 10 normal ovarian surface epithelial tissues) were collected by the Tissue Bank of the Obstetrics and Gynecology Hospital of Fudan University. And all samples were frozen immediately in liquid nitrogen and stored at $-80^{\circ} \mathrm{C}$ until analysis. None of these patients had received preoperative treatment. This study was approved by the Ethics Committee of the Obstetrics and Gynecology Hospital of Fudan University (No. [2017]82).

\section{Cell lines and cell cultures}

Three pairs of highly/poorly metastatic EOC cell lines (HEY-A8 and HEY, HO8910-PM and HO8910, and SKOV3-IP and SKOV3) were obtained from the University of Texas MD Anderson Cancer Center (Houston, TX, USA). The HEY-A8, HO8910-PM and SKOV3-IP cell lines are highly invasive EOC cell lines derived from the HEY, HO8910 and SKOV3 cell lines, respectively [13-19]. All cells were cultured in RPMI-1640 medium (Genom, Hangzhou, China) with $10 \%$ foetal bovine serum (FBS; Gibco, Grand, Island, $\mathrm{NY}$, USA), $100 \mathrm{U} / \mathrm{ml}$ penicillin and $100 \mu \mathrm{g} / \mathrm{ml}$ streptomycin at $37^{\circ} \mathrm{C}$ in a humidified atmosphere with $5 \%$ carbon dioxide.

\section{Establishment of stable AOC4P-knockdown and AOC4P- overexpression tumor cells}

The lentiviral vectors, which were used to knockdown or overexpress AOC4P, were constructed by Genechem (Shanghai, China). The recombinant AOC4P-siRNA-1, full length of AOC4P and the negative control lentivirus were prepared, all of which were labeled with luciferase (luc-lentivirus). After $72 \mathrm{~h}$, luciferin $(0.15 \mathrm{mg} / \mathrm{ml})$ was added into culture medium. To confirm luciferase expression of infected cells, they were then imaged under Bioluminescence Imaging Facility. Subsequently, positive cell clones were selected by puromycin for approximately 14 days to obtain the following new stable cell lines: HEYluc-NC, HEY-luc-KD, HEY-A8-luc-NC, HEY-A8-luc-OE, HO8910-PM-luc-NC, H08910-PM-luc-OE, SKOV3-IPluc-NC and SKOV3-IP-luc-OE.

\section{RNA extraction and quantitative real-time polymerase chain reaction (qRT-PCR)}

Total RNA was extracted with TRIZOL reagent (TAKARA, Code No.9109, Dalian, China) and the isolated RNA was reverse transcribed to cDNA utilizing the Prime-Script ${ }^{\text {tw }}$ RT Master Mix (TAKARA, RR036A, Dalian, China). The synthesized cDNA was subjected to qRT-PCR using SYBR Green qPCR (TAKARA, RR820A, Dalian, China). All reactions were performed according to the manufacturer's instructions. The expression of target genes relative to that of the control GAPDH was determined by the calculation $2^{-\Delta \Delta \mathrm{Ct}}$ method. The primer sequences are listed in Table 1.

\section{Cell transfection}

A negative control small interfering RNA (siRNA-NC) and AOC4P-siRNAs (GenePharma, Shanghai, China) were used in combination with Lipofectamine 3000 transfection reagent (Invitrogen, Carlsbad, CA, USA) to transfect HEY, HO8910, and SKOV3 cells based on the manufacturer's protocol. The target sequences of the AOC4P-siRNAs were 5'-GGUAAGGCAUCCUGGCAGAUU-3' (siRNA-1), 5' GGUCCUCUCUAUCUGUUUATT-3' (siRNA-2). The sequence of siRNA-NC was 5'-UUCUCCGAACGUGU CACGUTT-3'. Knockdown efficiency was assessed by qRT-PCR after $48 \mathrm{~h}$ of transfection.

\section{Scratch assay}

Seed the target cells on six-well plates. When the cells reached about $80-90 \%$ confluence, wounds were formed 
Table 1 Primers used in the study

\begin{tabular}{|c|c|c|}
\hline Gene & Primer & Sequence $\left(5^{\prime}-3^{\prime}\right)$ \\
\hline \multirow[t]{2}{*}{ GAPDH } & Forward & GTCATCAATGGAAATCCCATCA \\
\hline & Reverse & CCAG TGGACTCCACGACGTAC \\
\hline \multirow[t]{2}{*}{ AOC4P } & Forward & ACCAAGAGCCTGAAGACGA \\
\hline & Reverse & TGCCTGTCACCAGAACACTA \\
\hline \multirow[t]{2}{*}{ NONHSAT076754 } & Forward & AAGTTCTCACTCACCCACCTG \\
\hline & Reverse & GAAGCATGTACAGTTCAGCATGTG \\
\hline \multirow[t]{2}{*}{ IncTCF7 } & Forward & AGGAGTCCTTGGACCTGAGC \\
\hline & Reverse & AGTGGCTGGCATATAACCAACA \\
\hline \multirow[t]{2}{*}{ HEIRCC } & Forward & GCTGCTATTCTGGTGCCC \\
\hline & Reverse & TCAACTCCGATAAACAGGTGA \\
\hline \multirow[t]{2}{*}{ ZEB2NAT } & Forward & GAG AGA CGA GAG ACC CTG AA \\
\hline & Reverse & TGC ACA CAC CCT AAT ACA CAT \\
\hline \multirow[t]{2}{*}{ NKILA } & Forward & AACCAAACCTACCCACAACG \\
\hline & Reverse & ACCACTAAGTCAATCCCAGGTG \\
\hline \multirow[t]{2}{*}{ MMP2 } & Forward & GGAAGTCTGTGTTGTCCAGAGG \\
\hline & Reverse & TGATTGAAGCCAAGCGGTCT \\
\hline \multirow[t]{2}{*}{ MMP9 } & Forward & TCTACACCCAGGACGGCAAT \\
\hline & Reverse & GAAGCCGAAGAGCTTGTCCC \\
\hline \multirow[t]{2}{*}{ COL1A2 } & Forward & CTTGAAAGCCTCAAAAGTGT \\
\hline & Reverse & TCTCAGACCCAAGGACTATG \\
\hline \multirow[t]{2}{*}{ E-cadherin } & Forward & GACGCGGACGATGATGTGAAC \\
\hline & Reverse & TTGTACGTGGTGGGATTGAAGA \\
\hline \multirow[t]{2}{*}{ ZEB1 } & Forward & CCCTCTGGGATGCGAAACG \\
\hline & Reverse & CTGCTTCTAGACAGGAAATCCCAC \\
\hline
\end{tabular}

by draw a sterile $200 \mu \mathrm{L}$ pipette tip. The scraped cells were washed with phosphate-buffer-solution (PBS), and serum-free media were added. The scratch area was imaged at a certain time interval $(0 \mathrm{~h}, 24 \mathrm{~h}$ and $48 \mathrm{~h})$ and cell migration capacity was calculated using fomula as follow: cell mobility $=((1-$ corresponding point scratch width)/initial scratch width) $\times 100$.

\section{Cell migration assay}

Cell migration abilities were assessed using Transwell chambers with a pore size of $8 \mu \mathrm{m}$ (Millipore, Billerica, MA). The cells were loaded into the upper chamber with $200 \mu \mathrm{L}$ of serum-free RPMI-1640 medium. The bottom Transwell chambers were filled with $600 \mu \mathrm{L}$ of complete RPMI-1640 medium containing 10\% FBS. $24 \mathrm{~h}$ later, the cells on the lower surface were fixed, stained and then five random fields $(100 x)$ were counted under a microscope.

\section{Cell invasion assay}

The invasion assays were identical to the migration assay except that the Transwell membranes were pre-coated with Matrigel (BD Biosciences, Franklin Lakes, NJ, USA) as a matrix barrier before the cell incubation.
In vivo intraperitoneal metastasis model

The protocols were approved by the Institutional Animal Care and Use Committee of Fudan University. Female 5to 6-week-old Balb/c nude mice were obtained from Slac Laboratory Animal Co. Ltd. (Shanghai, China), maintained under a specific-pathogen free (SPF) condition and randomly distributed into two groups, the control group and the experimental knockdown group (six mice per group). The mice in the control group were intraperitoneally (i.p.) injected with HEY-luc-NC cells, while the mice in the knockdown group were injected with HEYluc-KD cells $(2 \times 106 / 0.2 \mathrm{ml})$. Then, the mice were monitored weekly at the Small Animal Bioluminescence Imaging Facility. Before imaging, each mouse was i.p. injected with luciferin $(150 \mathrm{mg} / \mathrm{kg})$ to stimulate luminescence. After 4 weeks, all mice were sacrificed, and the tumours were dissected for analysis.

\section{Tumour EMT RT-PCR array}

After HEY cells were transfected with AOC4P-siRNA-1 or AOC4P-NC for $24 \mathrm{~h}$, total RNA was extracted and then reverse transcribed into cDNA using an $\mathrm{RT}^{2}$ First Strand kit (Qiagen, Mississauga, ON, Canada). Based on the manufacturer's protocol, a cDNA library was established and we analyzed the reverse transcribed cDNA with a Human Tumour Metastasis RT2 Profiler ${ }^{\mathrm{Tm}}$ PCR array (Qiagen, Mississauga, ON, Canada), which contained 84 well known genes involved in the tumour EMT process. The expression fold-changes were calculated with the $2^{-\Delta \Delta \mathrm{Ct}}$ method, and the results are exhibited.

\section{Western blot (WB) analysis}

WB analysis was conducted as described previously [20]. The primary antibodies used were rabbit anti-GAPDH (Cell Signaling Technology, \#5174, USA), anti-MMP9 (Abcam, ab76003, Cambridge, UK) and anti-COL1A2 (Abcam, ab, Cambridge, UK) antibodies.

\section{Data analysis}

The data analysis was conducted using SPSS version 20.0 software (SPSS, Inc., Chicago, IL, USA) and GraphPad Prism software (Prism 5.0, GraphPad Software, San Diego, CA, USA). Continuous data were analyzed by Student's t-test (2 groups) or one-way ANOVA ( $>2$ groups), while categorical data were analyzed by the $\chi^{2}$ test or Fisher's exact test. $P<0.05$ was defined as statistically significant.

\section{Results}

Downregulation of AOC4P expression is linked to advanced and aggressive $\mathrm{OC}$ clinical characteristics

Based on existing studies [10, 11, 21-28], we chose six EMT-associated lncRNAs and investigated whether they 
are involved in EOC. Using highly metastatic EOC cell lines (HEY-A8, HO8910-PM, and SKOV3-IP) and their parental poorly metastatic sublines (HEY, HO8910, and SKOV3) as a cell model, we observed that AOC4P displayed an optimal consistency in expression levels and exhibited significantly lower levels in the highly metastatic cell lines than in the parental cell lines (Fig. 1a). Therefore, AOC4P were the focus of further clinical and functional explorations.

To further investigate the clinical implications of AOC4P in EOC, we tested AOC4P expression in 70 EOC and 10 normal ovarian surface epithelial tissues samples by qRT-PCR. The results showed that the AOC4P expression in the EOC tissue samples was significantly lower than that in the normal tissues samples (Fig. 1b). The expression levels of AOC4P in the lymph node metastasis-positive group (lymph node $(+)$ ) were significantly lower than those in the lymph node metastasis-negative group (lymph node (-)) (Fig. 1c). Additionally, relatively lower AOC4P expression levels were detected in the advanced FIGO stage (III-IV) cancer samples than in the early FIGO stage (I-II) samples (Fig. 1d). Moreover, to analyze the clinicopathological significance of AOC4P expression in EOC, the $70 \mathrm{EOC}$ patients were divided into two groups, i.e., a high
AOC4P expression group $(n=35)$ and a low AOC4P expression group $(n=35)$ based on the median value of relative AOC4P expression. Consequently, low AOC4P expression was positively associated with FIGO stage and lymph node metastasis, but no significant difference in other clinicopathological parameters such as age, histological subtype, residual tumour diameter, CA125 expression and ascites, were found (Table 2). These results illustrated that AOC4P has the potential to function as a tumour suppressor and might have an essential impact on EOC metastasis and aggressiveness.

\section{AOC4P interference facilitates EOC cell metastasis in vitro} Given that higher AOC4P expression was observed in the poorly metastatic EOC cell lines (HEY, HO8910, and SKOV3) (Fig. 1a), as well as the fact that lower expression levels of AOC4P were associated with aggressive tumour phenotypes in EOC, we speculated that AOC4P might exert a crucial function in EOC cell migratory and invasive abilities. To verify this hypothesis, a series of functional experiments in selected cells were conducted based on the siRNA-mediated knockdown of AOC4P expression. Initially, the interference efficiency of AOC4P expression was determined by qRT-PCR (Fig. 2a). Subsequently, tumour cell metastatic potential was evaluated in AOC4P-siRNA-

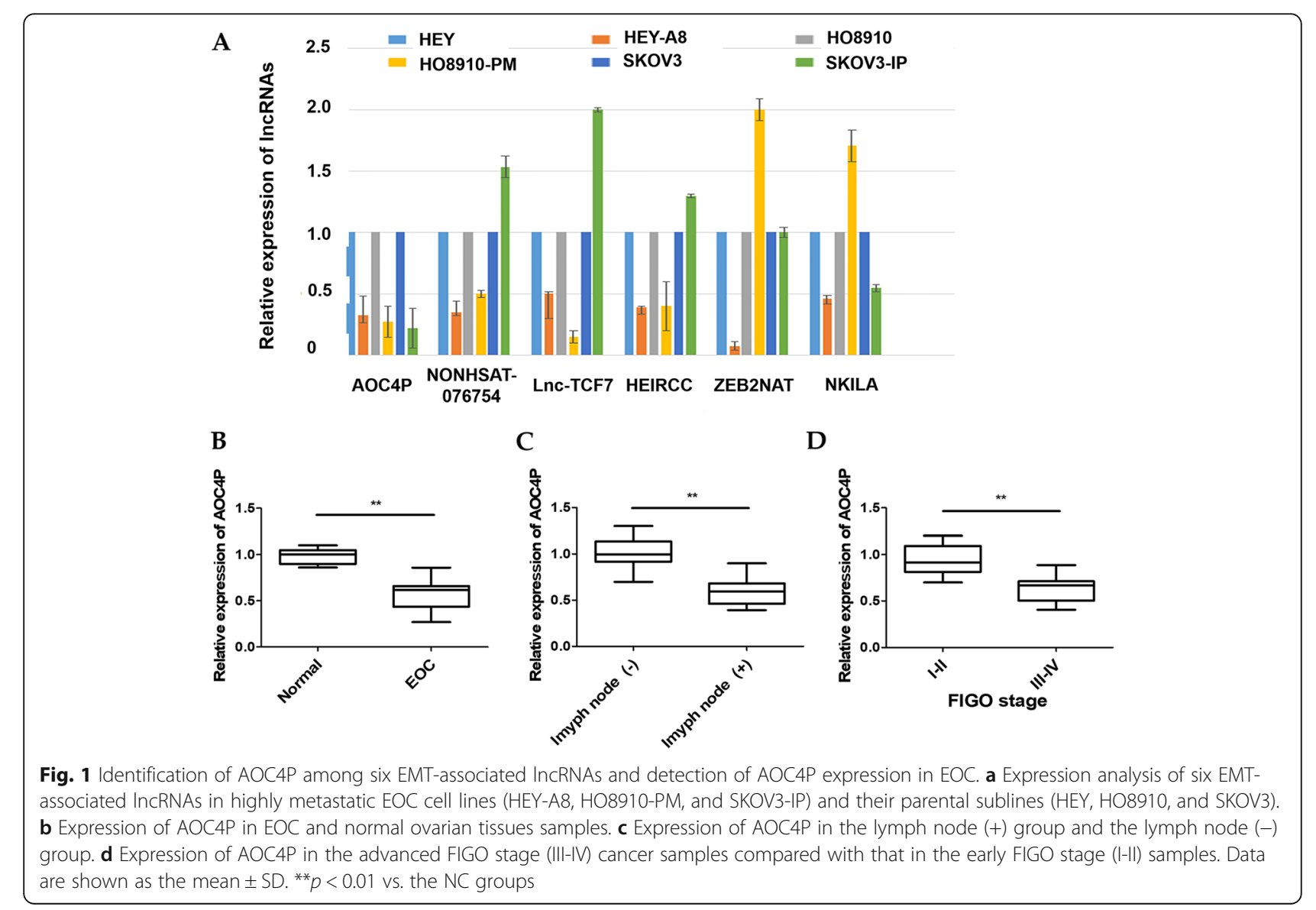


Table 2 Correlation of AOC4P expression with clinicopathological characteristics in 70 EOC patients

\begin{tabular}{|c|c|c|c|}
\hline \multirow[t]{2}{*}{ Variable } & $\begin{array}{l}\text { Low AOC4P } \\
\text { expression }(n=35)\end{array}$ & $\begin{array}{l}\text { High AOC4P } \\
\text { expression }(n=35)\end{array}$ & \multirow[t]{2}{*}{$P$-value } \\
\hline & $n(\%)$ & \multirow{2}{*}{\multicolumn{2}{|c|}{$n(\%)$}} \\
\hline \multicolumn{2}{|c|}{ Age (years) } & & \\
\hline$<50$ & $14(40.0)$ & $10(28.6)$ & \\
\hline$\geq 50$ & $21(60.0)$ & $25(71.4)$ & 0.314 \\
\hline \multicolumn{4}{|c|}{ Histological subtype } \\
\hline Serous & $26(80.0)$ & $23(62.9)$ & \\
\hline Others & $9(20.0)$ & $12(37.1)$ & 0.434 \\
\hline \multicolumn{4}{|c|}{ FIGO Stage } \\
\hline$|-| \mid$ & $2(5.7)$ & $8(22.9)$ & \\
\hline III-IV & $33(94.3)$ & $27(77.1)$ & 0.040 \\
\hline \multicolumn{4}{|c|}{ Residual tumour diameter (cm) } \\
\hline$<1$ & $32(91.4)$ & $28(80.0)$ & \\
\hline$\geq 1$ & $3(8.6)$ & $7(20.0)$ & 0.172 \\
\hline \multicolumn{4}{|c|}{ Lymph node metastasis } \\
\hline Absent & $7(20.0)$ & $27(77.1)$ & \\
\hline Present & $28(80.0)$ & $8(22.9)$ & $<0.001$ \\
\hline \multicolumn{4}{|c|}{ CA125 level (U/ml) } \\
\hline$<600$ & $10(28.6)$ & $12(34.3)$ & \\
\hline$\geq 600$ & $25(71.4)$ & $23(65.7)$ & 0.607 \\
\hline \multicolumn{4}{|l|}{ Ascites } \\
\hline$<100$ & $19(54.3)$ & $24(68.6)$ & \\
\hline$\geq 100$ & $16(45.7)$ & 11 (31.4) & 0.220 \\
\hline
\end{tabular}

transfected cells with Transwell and scratch assays. The results revealed that AOC4P interference remarkably facilitates the metastasis of HEY, HO8910, and SKOV3 cells in vitro (Fig. 2b-d).

\section{Overexpression of AOC4P inhibits EOC cell metastasis in vitro}

Since the loss-of-function assays determined that the knockdown of AOC4P expression facilitated EOC cell metastasis in vitro, we further investigated whether the overexpression of AOC4P would have the opposite effect on highly metastatic EOC cell lines (HEY-A8, HO8910PM, and SKOV3-IP). We constructed stable AOC4P overexpression EOC cell lines (Fig. 3a) and, as expected, observed that overexpression of AOC4P significantly attenuated the metastasis of HEY-A8, HO8910-PM and SKOV3-IP cells in vitro (Fig. 3b-d). Collectively, these results indicated that AOC4P could inhibit EOC cell metastasis in vitro.

\section{AOC4P represses EOC metastasis in vivo}

To investigate the metastatic role of AOC4P in vivo, a nude mouse model of abdominal metastasis was established. The results gained from bioluminescence imaging showed that compared to that of HEY-luc-NC group, the luciferase signals of the HEY-luc-KD group were increased (Fig. 4a). The significant difference in photon flux quantification between the two groups was found early at 14 days after the abdominal injection and became more obvious at 21 and 28 days (Fig. 4b). Data analysis of the tumours dissected after 4 weeks revealed that the HEY-luc-KD group developed multiple visible metastatic nodules in the abdominal cavity, including abdominal wall lesions, omental lesions, mensenteric lesions, uterine lesions, subphrenic lesions, hepatic metastatic nodular lesions and posterior peritoneal lesions. In comparison, the HEY-luc-NC group exhibited limited tumour formation at the primary injection site in the abdominal wall or in the omental area near the injection site (Fig. 4c). Statistically, the HEY-luc-KD group showed more and larger metastatic nodules than the HEYluc-NC group (Fig. 4d). Altogether, these results indicated that AOC4P could suppress EOC metastasis in vivo.

\section{AOC4P affects EOC metastasis via EMT-mediated gene alterations in MMP9 and COL1A2}

Since both the in vitro and in vivo data supported the anti-metastatic role of AOC4P in EOC, we explored the potential mechanisms involved. Interestingly, through observation of AOC4P-siRNA-transfected cells, we found that the knockdown of AOC4P resulted in the cells exhibiting a spindle-shape morphology and (or) starting to separate from one another, while the cells in the NC groups retained their morphology and cell-cell contact (Fig. 5a). Based on these phenomena, we hypothesized that EMT might participate in AOC4P-induced anti-metastatic activity in EOC. Initially, we performed an EMT RT-PCR array to explore whether the expression levels of certain EMT-related genes were changed after AOC4P interference. As a result, five genes were remarkably dysregulated ( $\geq 2$-fold), with four genes (MMP2, MMP9, COL1A2, and ZEB1) upregulated and one gene (E-cadherin) downregulated (Table 3). Then, qRT-PCR and WB assays confirmed that MMP9 and COL1A2 was upregulated at both the mRNA and protein levels in two AOC4P-siRNA-transfected cells lines (HEY and HO8910) (Fig. 5b-c). Collectively, these data revealed that the two EMT-related genes MMP9 and COL1A2 might be involved in the AOC4P-mediated anti-metastatic effect.

\section{Discussion}

EOC is recognized as the most malignant subtype of OC with uncomfortably poor prognosis [29]. The high rate of metastasis is acknowledged as chief culprit contributing to the poor outcome of EOC. Evidence indicates that EMT is one of the major molecular mechanisms 

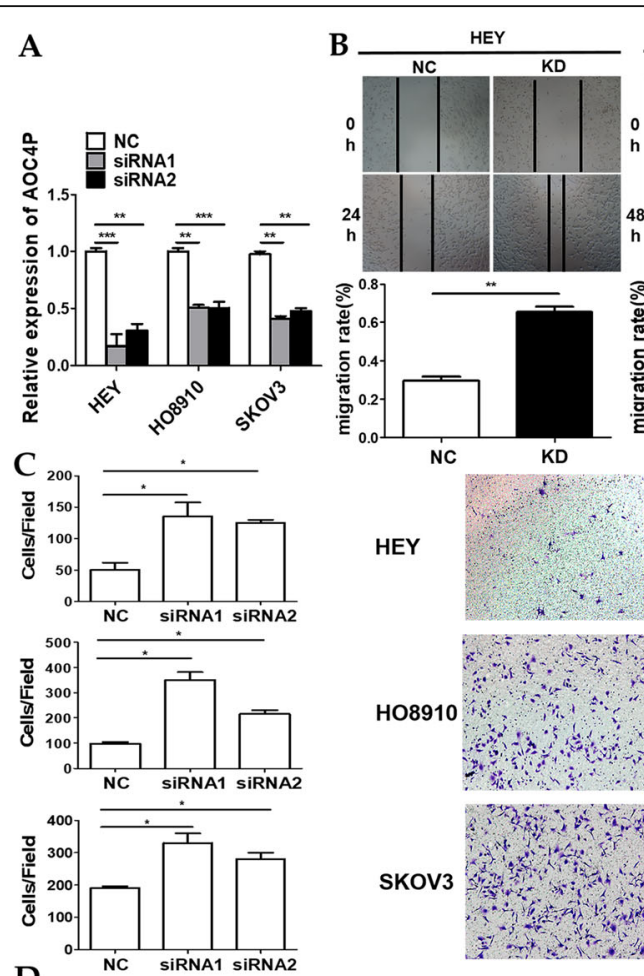

D
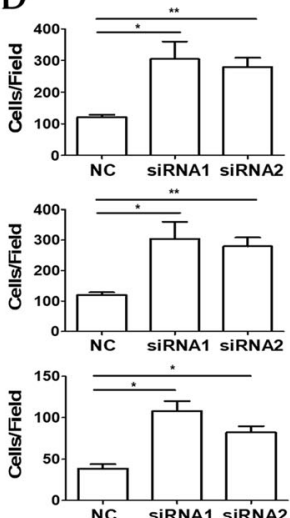
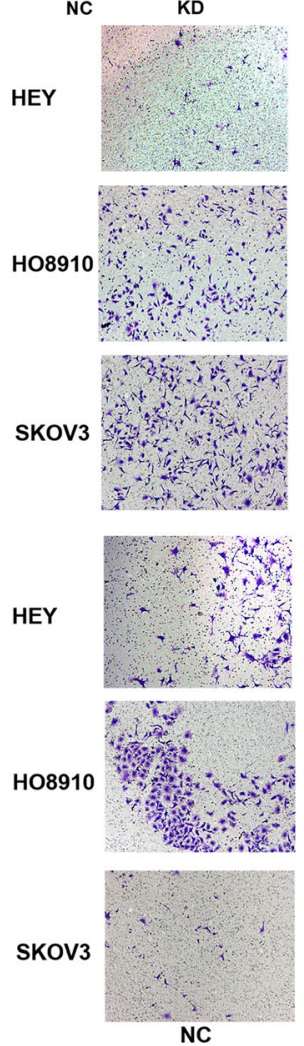
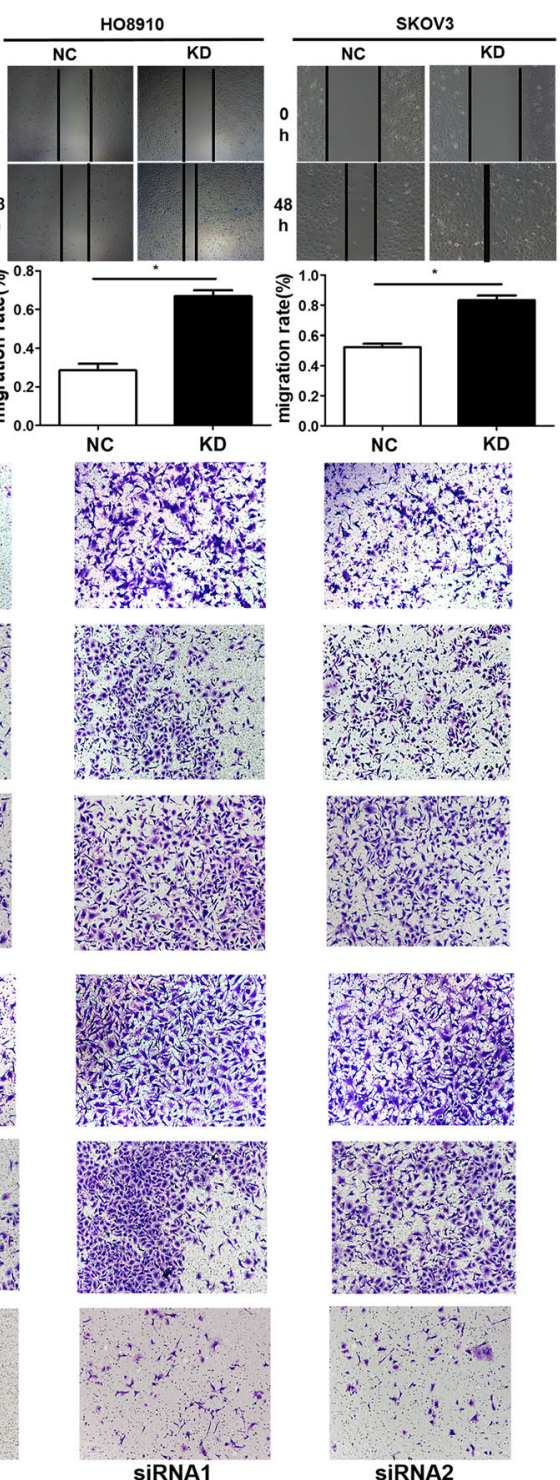

Fig. 2 Knockdown of AOC4P promotes migration and invasion in HEY, HO8910 and SKOV3 cells. a The interference efficiency of AOC4P expression was determined by qRT-PCR. b Cell migration activities after AOC4P interference were assessed by wound healing assays. $\mathbf{c}$ Transwell migration assays were conducted to confirm the cell migration activity. $\mathbf{d}$ Cell invasion activities after AOC4P interference were assessed by transwell assays. Data are shown as the mean \pm SD. ${ }^{*} p<0.05$, ${ }^{* *} p<0.01$, and ${ }^{* *} p<0.001$ vs. the NC groups

inducing cancer metastasis [30] and is also an essential event in initiating metastasis of malignant tumours [31].

Mounting studies have elucidated the role of lncRNAs in a wide variety of human malignancies. In EOC, an increasing numbers of lncRNAs have been demonstrated to be related to metastasis, and several of these lncRNAs are considered to be EMT-related lncRNAs (e.g., HOTAIR, UCA1, MALAT1, CCAT1, Linc-ROR, H19, and NEAT1) [9]. Despite these advances, research on EMT-related lncRNAs has been limited in EOC. Therefore, it is worth gaining insight into novel EMT-related molecules, which may provide novel perspective for EOC diagnosis and therapy.
Using the literature, we identified six EMT-related lncRNAs (AOC4P, NONHSAT076754, Lnc-TCF7, HEIRCC, ZEB2NAT and NKILA), whose roles in EOC had not been investigated. Among them, AOC4P gained the most attention because the expression of AOC4P was consistently significantly lower in our highly metastatic cell lines (HEY-A8, HO8910-PM, and SKOV3-IP) than in the parental poorly metastatic cell lines (HEY, HO8910, and SKOV3). AOC4P, a recently identified lncRNA, was initially reported to suppress invasion and metastasis in patients with HCC [10]. Subsequently, it was reported that decreased expression of AOC4P was closely correlated with metastatic event and 

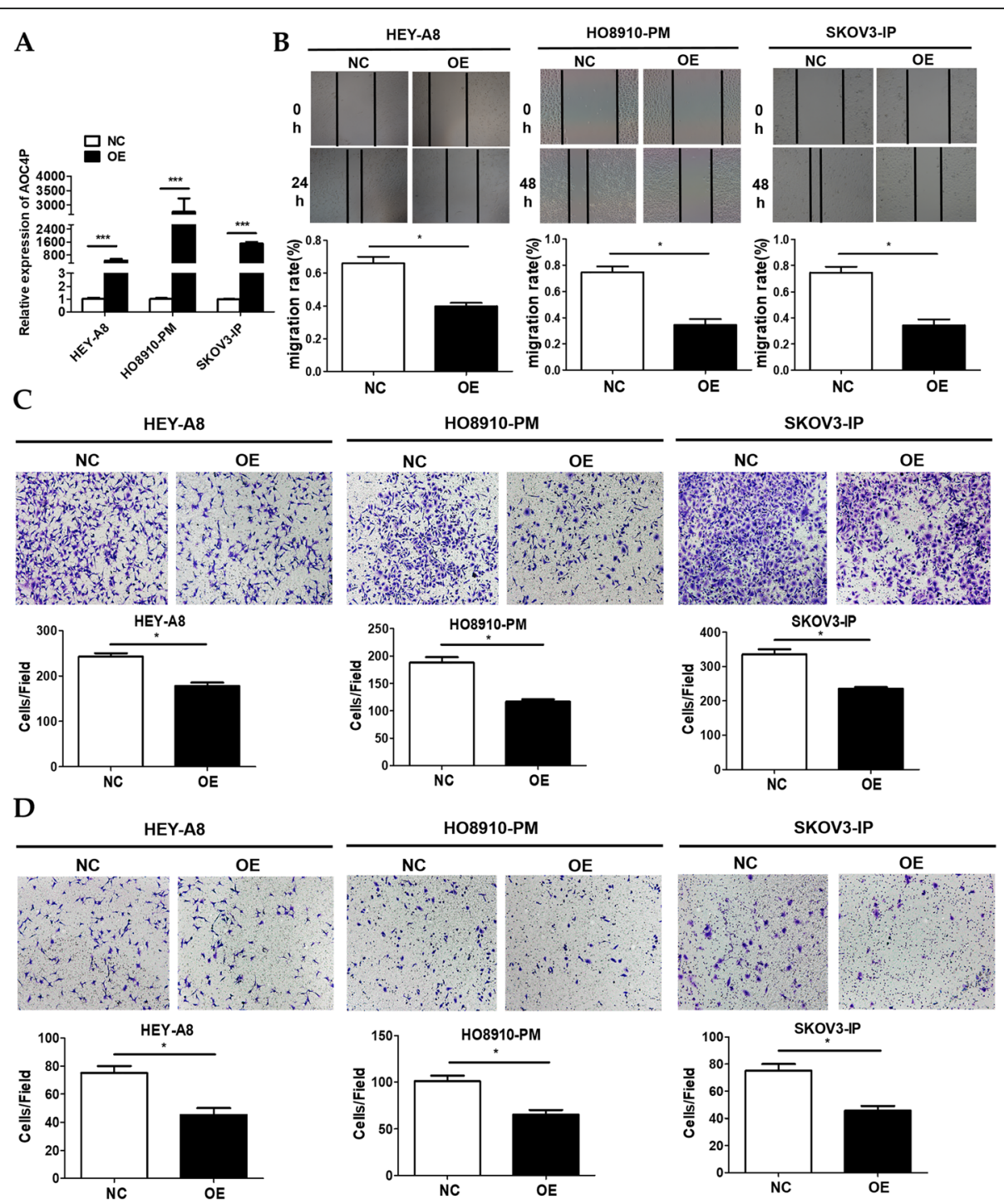

Fig. 3 Overexpression of AOC4P hinders EOC cell metastasis in HEY-A8, HO8910-PM and SKOV3-IP cells. a Efficiency of AOC4P overexpression was confirmed by qRT-PCR. b Migration of AOC4P -overexpression cells was evaluated by wound healing assays. $\mathbf{c}$ Transwell migration assays were conducted to confirm the cell migration activity. $\mathbf{d}$ Invasion of AOC4P -overexpression cells was detected by transwell invasion assays. Data are shown as the mean \pm SD. ${ }^{*} p<0.05$, and ${ }^{* *} p<0.001$ vs. NC groups

poor prognosis in patients with CRC [11]. Evidence also supported that AOC4P regulated tumor cell proliferation and invasion in GC [12]. However, no study has illustrated the involvement of AOC4P in EOC. Here, we found that the de-regulated expression of AOC4P was linked to aggressive clinicopathological variables including advanced FIGO stage and lymph node metastasis, suggesting that AOC4P might act as a tumour suppressor and could be applied as an additional biomarker in distinguishing less malignant and metastasis-prone outcome in EOC.

Recently, several lncRNAs have been identified to have downregulated expression and act as suppressive factors during the progression of multiple types of cancers. For instance, the silencing of SPRY4-IT1 leads OC cells to develop a more aggressive phenotype [32]. Low NKILA expression has been implicated in tongue squamous cell carcinoma invasion and metastasis [28]. For AOC4P, previous studies have revealed that the overexpression of AOC4P prominently reduced HCC and CRC cell metastasis. However, in GC, AOC4P was upregulated and promoted the migration and invasion of GC cells [10-12]. There was previous evidence that the expression levels of lncRNAs varied in various carcinomas with different tumor cell functions. For example, lncRNA BANCR was expressed differentially in GC and colon cancer [33, 34]. Inspired by these studies combined with our findings that decreased expression of AOC4P was correlated with lymph node metastasis and that tissue samples from the 


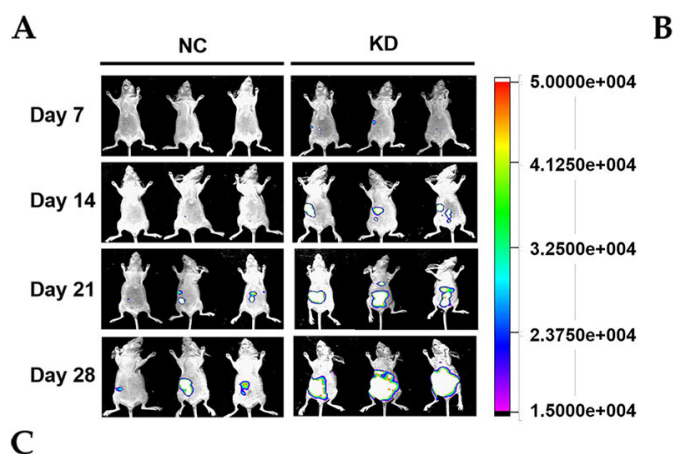

B
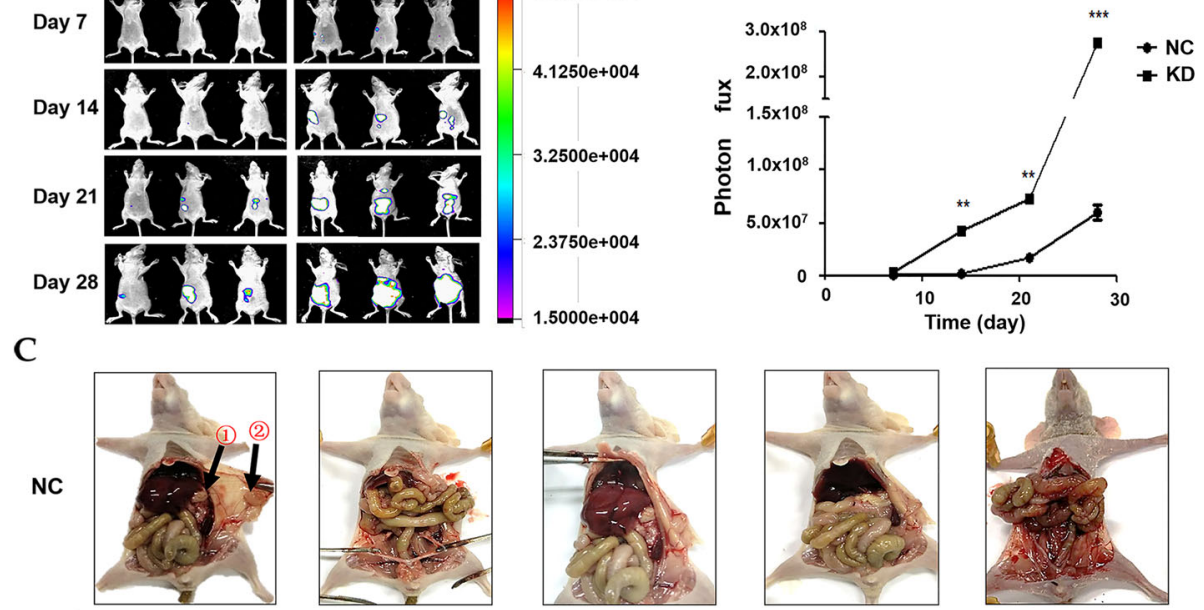

(1) Omental lesions

(2) Abdominal wall lesions

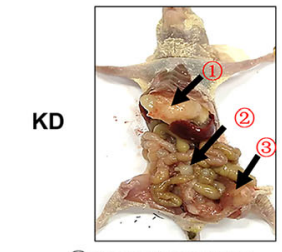

(1) Omental lesions (2) Mensentery lesions

(3) Abdominal wall lesions

D

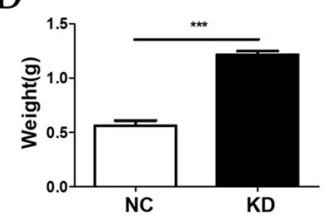

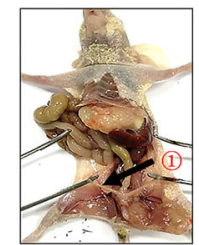

(1) Uterine lesions

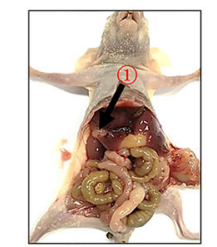

(1) Subphrenic lesions
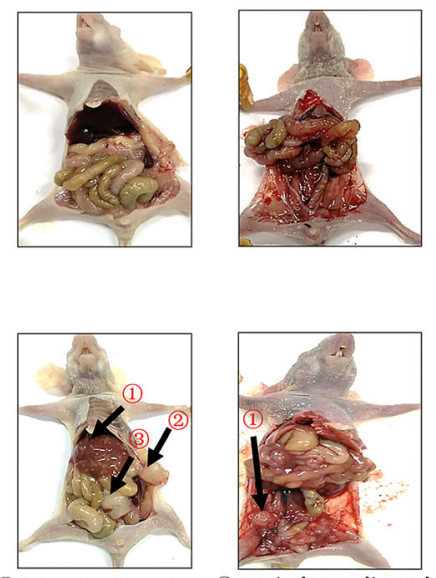

(1) Hepatic metastatic (1) Posterior peritoneal nodular lesions lesions

(2) Abdominal wall lesions

(3) Mensentery lesions
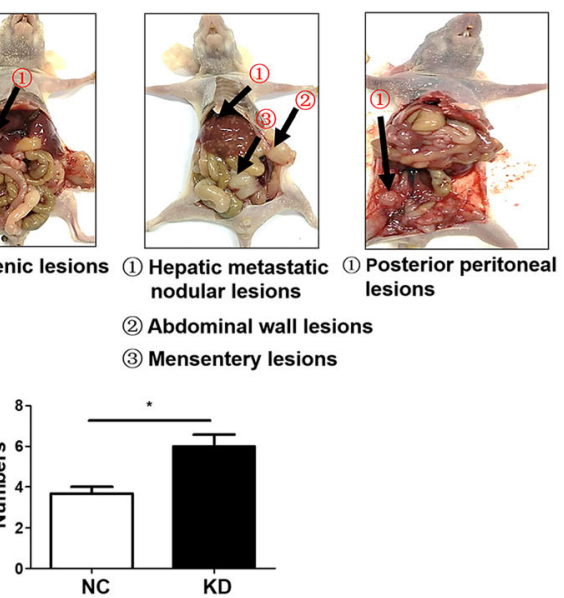

Fig. 4 AOC4P suppresses metastasis in vivo. a Representative images of the luciferase signals in the HEY-luc-KD and HEY-luc-NC groups. b Quantification of the photon flux from the lesions detected by bioluminescence imaging. c Representative views of visible metastases in the peritoneal cavity. $\mathbf{d}$ Analysis of the numbers and weights of dissected tumours. Data are shown as the mean \pm SD. ${ }^{*} p<0.05,{ }^{* *} p<0.01$, and ${ }^{* * *}$ $p<0.001$ vs. the NC groups

lymph node metastasis-positive group expressed lower AOC4P levels than those from the corresponding lymph node metastasis-negative group, we attempted to ascertain whether AOC4P was involved in EOC metastasis. As anticipated, Transwell and scratch assays showed that the depletion of AOC4P could promote migration and invasion in poorly metastatic EOC cell lines. In contrast, the overexpression of AOC4P impaired the metastatic and invasive potential of highly metastatic EOC cell lines in vitro. In line with the results of the in vitro assays, an in vivo experiment indicated that silencing AOC4P led to a remarkable increase in the number, weight and distribution of the tumour. These results, similar to its functions in HCC, indicate that AOC4P acts as a critical suppressor in EOC metastasis.
To date, the mechanisms underlying lncRNA-involved metastasis have not been fully elucidated. It is wellacknowledged that EMT is a critical initiating factor of tumour metastasis and invasion. Growing evidence has noted that EMT-related lncRNAs play critical roles in the progression of various types of tumours via diverse mechanisms [35]. Previous in mechanistic investigations have indicated that certain EMT regulators participate in AOC4P-mediated anti-metastatic behaviours [10-12]. Nonetheless, the molecular events underlying AOC4Pmediated anti-metastatic behavior of EOC are not elucidated. In the current study, we found that AOC4Pknockdown cells experienced morphological and distribution transformations from a cobblestone-like shape and (or) tight adhesion to spindle-shape morphology 


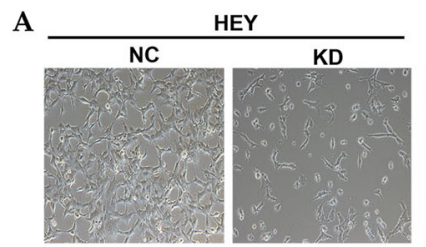

B
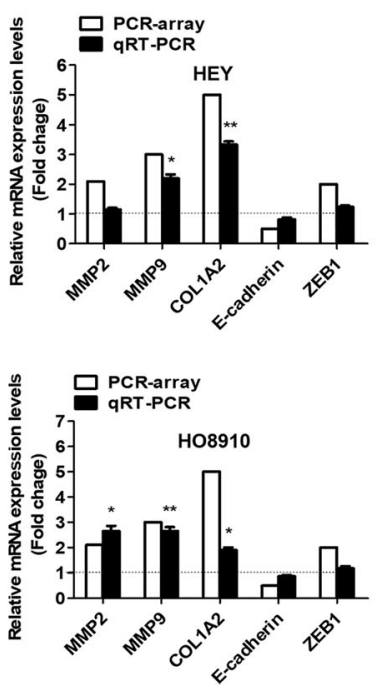

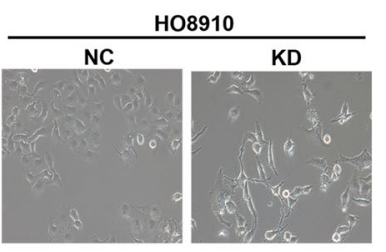

C
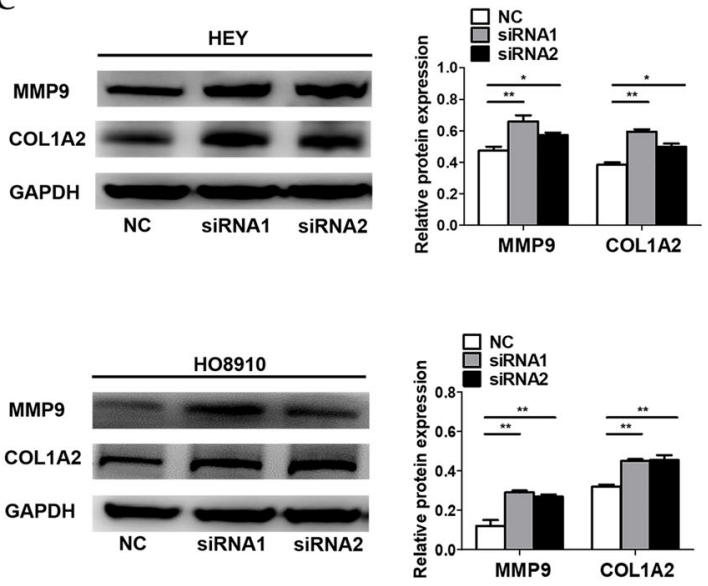

Fig. 5 AOC4P suppresses EOC metastasis via the EMT-regulated downstream genes MMP9 and COL1A2. a Representative images of the morphology of the cells in AOC4P-siRNA or siRNA-NC groups are shown. $\mathbf{b}$ The expression levels of MMP9 and COL1A2 were detected by qRTPCR in SKOV3 and HO8910 cells transfected with AOC4P-siRNA or siRNA-NC. c WB verification of MMP9 and COL1A2 expression in SKOV3 and HO8910 cells transfected with AOC4P-siRNA or siRNA-NC. Data are shown as the mean \pm SD. * $p<0.05$, and ${ }^{* *} p<0.01$ vs. the NC groups

and (or) scattered cell-cell adhesion and these results agree with EMT features. Then, a tumour EMT RT-PCR array indicated that certain differentially expressed genes were identified after the knockdown of AOC4P expression. The subsequent verification of the results from the array confirmed that the expression of mesenchymalassociated factors including MMP9 and COL1A2 was upregulated. The changes in expression were validated at both the mRNA and protein levels, which further demonstrated that the EMT process is involved in AOC4P-mediated metastasis inhibition. Notably, certain EMT markers, such as E-cadherin, $\mathrm{N}$-cadherin, vimentin, did not display significant difference in our study. Especially, the expression of E-cadherin was downregulated in data from tumour EMT RT-PCR array while it showed no difference in qRT-PCR verification in both two tumor cells (HEY and HO8910). According to the literature, there is evidence that the expression of Ecadherin displays no difference in several subtypes of OC [36]. In addition, between normal HCC cells and AOC4P-overexpressing HCC cells, there is no difference in E-cadherin expression [10]. The fact that we set the cut-off value of fold change as 2 when we analyzed the data from EMT PCR array, resulted in some differentially expressed EMT-related genes with fold change lower than 2 were ignored. Besides, although some typical EMT markers showed no difference in mRNA level in this manuscript, the protein level of these genes might be altered by the steps after transcription including posttranscriptional, translation and post-translational modification. In future work, WB assays are essential to be performed on these typical EMT markers to determine

Table 3 Differential expression of genes ( $\geq 2$-fold) between HEY-AOC4P-siRNA-1 cells and HEY-siRNA-NC cells as identified by an EMT array

\begin{tabular}{lllll}
\hline Gene name & GeneBank ID & Description & Function & Fold change \\
\hline MMP2 & NM_005343 & Matrix metallopeptidase 2 & Mesenchymal marker & 2.1 \\
MMP9 & NM_004994 & Matrix metallopeptidase 9 & Mesenchymal marker & 3.3 \\
COL1A2 & NM_000089 & collagen type I alpha 2 chain & Mesenchymal marker & 5.1 \\
E-cadherin & NM_004360 & Cadherin 1, type 1, E-cadherin (epithelial) & Epithelial marker & -2.0 \\
ZEB1 & NM_030751 & Zinc finger E-box binding homeobox 1 & Mesenchymal marker & 2.0 \\
\hline
\end{tabular}


if they are differently expressed in protein level. Admittedly, there are some limitations of present study. First, adequate paraffin-embedded clinical tissue samples, which were not available in present study due to the limitation of our Tissue Bank, are needed for in situ hybridization (ISH) assays to further evaluate the expression and localization of AOC4P and better reveal the relevance of AOC4P to EOC. Second, more comprehensive and detailed work needs to be done to better unveil the biological processes AOC4P participated in, downstream genes AOC4P induced and the direct or indirect relationship between AOC4P and downstream genes.

\section{Conclusion}

In conclusion, this study collected comprehensive data from the clinic, function and mechanism, and offers the first evidence of the role of AOC4P in EMT-involved metastasis in EOC. We show that the under-expression of AOC4P underlies the onset of EMT and aggressive metastasis in EOC by upregulating the expression of MMP9 and COL1A2. We have also shown that low expression levels of AOC4P are positively related with an advanced tumour stage and lymph node metastasis in EOC, suggesting that AOC4P could serve as an effective target for anti-metastatic strategies in EOC.

\section{Acknowledgements}

Not applicable.

\section{Authors' contributions}

Data curation, Xiaojing Lin and Xiaoyan Tang; Formal analysis, Xiaojing Lin and Tingting Zheng; Funding acquisition, Keqin Hua; Investigation, Junjun Qiu and Keqin Hua; Methodology, Junjun Qiu; Project administration, Xiaojing Lin, Xiaoyan Tang and Junjun Qiu; Supervision, Keqin Hua; Writing original draft, Xiaojing Lin; Writing - review \& editing, Junjun Qiu and Keqin Hua. The author(s) read and approved the final manuscript.

\section{Funding}

This project was supported by funding from the National Natural Science Foundation of China (No. 81971361; to Jun-jun Qiu), the Natural Science Foundation of Shanghai Science and Technology (No. 19ZR1406900; to Jun-jun Qiu), and shanghai "Rising Stars of Medical Talent" Youth Development Program to Jun-jun Qiu, the Research and Innovation Project of Shanghai Municipal Education Commission (No. 2019-01-07-00-07-E00050; to Ke-qin Hua), and the artificial Intelligence Innovation Project of Shanghai Municipal Commission of Economy and Informatization (No. 2018-RGZN-02041; to Ke-qin Hua).

\section{Availability of data and materials}

Not applicable.

\section{Ethics approval and consent to participate}

This study was approved by the Ethics Committee of the Obstetrics and Gynecology Hospital of Fudan University (No. [2017]82) and written informed consent was obtained for the use of tissue samples from all participants enrolled in this study.

\section{Consent for publication}

Not applicable.

\section{Competing interests}

The authors declare that they have no competing interests.

\section{Author details}

'Department of Gynecology, Obstetrics and Gynecology Hospital of Fudan University, 419 Fangxie Road, Shanghai 200011, P.R. China. ${ }^{2}$ Reproductive Medicine Center, The First Affiliated Hospital of Wenzhou Medical University, Wenzhou 325000, P.R. China. ${ }^{3}$ Shanghai Key Laboratory of Female Reproductive Endocrine-Related Diseases, Fudan University, Shanghai 200011, P.R. China.

Received: 29 August 2019 Accepted: 3 April 2020

Published online: 25 April 2020

\section{References}

1. Morgan RJ Jr, Alvarez RD, Armstrong DK, Boston B, Burger RA, Chen LM, et al. Epithelial ovarian cancer. J Natl Compr Canc Netw. 2011;9:82-113.

2. Yang D, Sun Y, Hu L, Zheng H, Ji P, Pecot CV, et al. Integrated analyses identify a master microRNA regulatory network for the mesenchymal subtype in serous ovarian cancer. Cancer Cell. 2013;23:186-99.

3. Rustin GJ, van der Burg ME, Griffin CL, Guthrie D, Lamont A, Jayson GC, et al. Early versus delayed treatment of relapsed ovarian cancer (MRC OV05/ EORTC 55955): a randomised trial. Lancet. 2010;376:1155-63.

4. Vanharanta S, Massague J. Origins of metastatic traits. Cancer Cell. 2013;24: 410-21.

5. Cao L, Shao M, Schilder J, Guise T, Mohammad KS, Matei D. Tissue transglutaminase links TGF-beta, epithelial to mesenchymal transition and a stem cell phenotype in ovarian cancer. Oncogene. 2012;31:2521-34.

6. Vergara D, Merlot B, Lucot JP, Collinet P, Vinatier D, Fournier I, et al. Epithelialmesenchymal transition in ovarian cancer. Cancer Lett. 2010;291:59-66.

7. Kalluri R, Weinberg RA. The basics of epithelial-mesenchymal transition. J Clin Invest. 2009;119:1420-8.

8. Moustakas A, Heldin CH. Signaling networks guiding epithelialmesenchymal transitions during embryogenesis and cancer progression. Cancer Sci. 2007;98:1512-20.

9. Lin X, Qiu J, Hua K. Long non-coding RNAs as emerging regulators of epithelial to mesenchymal transition in gynecologic cancers. Bioscience trends. 2018;12:342-53.

10. Wang TH, Lin YS, Chen Y, Yeh $C T$, Huang YL, Hsieh TH, et al. Long noncoding RNA AOC4P suppresses hepatocellular carcinoma metastasis by enhancing vimentin degradation and inhibiting epithelial-mesenchymal transition. Oncotarget. 2015;6:23342-57.

11. Kang J, Li Z, Kong FH, Zhang M, Chang H. Overexpression of IncRNA AOC4P indicates a poor prognosis for colorectal cancer and regulates cell metastasis by epithelial mesenchymal transition. Int J Clin Exp Pathol. 2017;10:1840-6.

12. Zhang K, Lu C, Huang X, Cui J, Li J, Gao Y, et al. Long noncoding RNA AOC4P regulates tumor cell proliferation and invasion by epithelial-mesenchymal transition in gastric cancer. Ther Adv Gastroenterol. 2019;12:1756284819827697.

13. Bai F, Feng J, Cheng Y, Shi J, Yang R, Cui H. Analysis of gene expression patterns of ovarian cancer cell lines with different metastatic potentials. Int J Gynecol Cancer. 2006;16:202-9.

14. Lehtinen J, Raki M, Bergstrom KA, Uutela P, Lehtinen K, Hiltunen A, et al. Pre-targeting and direct immunotargeting of liposomal drug carriers to ovarian carcinoma. PLoS One. 2012;7:e41410.

15. Li J, Liang $S, Y u H$, Zhang J, Ma D, Lu X. An inhibitory effect of miR-22 on cell migration and invasion in ovarian cancer. Gynecol Oncol. 2010;119:543-8.

16. Shenhua X, Lijuan Q, Hanzhou N, Xinghao N, Chihong Z, Gu Z, et al. Establishment of a highly metastatic human ovarian cancer cell line (HO8910PM) and its characterization. J Exp Clin Cancer Res. 1999;18:233-9.

17. Wang $Y$, Dong $L$, Cui $H$, Shen DH, Wang Y, Chang XH, et al. Up-regulation of mitochondrial antioxidation signals in ovarian cancer cells with aggressive biologic behavior. J Zhejiang Univ Sci B. 2011;12:346-56.

18. Xu W, Mezencev R, Kim B, Wang L, McDonald J, Sulchek T. Cell stiffness is a biomarker of the metastatic potential of ovarian cancer cells. PLoS One. 2012;7:e46609.

19. Yu D, Wolf JK, Scanlon M, Price JE, Hung MC. Enhanced c-erbB-2/neu expression in human ovarian cancer cells correlates with more severe malignancy that can be suppressed by E1A. Cancer Res. 1993;53:891-8.

20. Qiu JJ, Lin YY, Ye LC, Ding JX, Feng WW, Jin HY, et al. Overexpression of long non-coding RNA HOTAIR predicts poor patient prognosis and promotes tumor metastasis in epithelial ovarian cancer. Gynecol Oncol. 2014;134:121-8.

21. Li T, Zhu J, Zuo S, Chen S, Ma J, Ma Y, et al. 1,25(OH)2D3 attenuates IL1 beta-induced epithelial to mesenchymal transition through inhibiting the expression of IncTCF7. Oncol Res. 2019;27:739-50. 
22. Wu B, Chen M, Gao M, Cong Y, Jiang L, Wei J, et al. Down-regulation of IncTCF7 inhibits cell migration and invasion in colorectal cancer via inhibiting TCF7 expression. Hum Cell. 2019;32:31-40.

23. Wu J, Zhang J, Shen B, Yin K, Xu J, Gao W, et al. Long noncoding RNA InCTCF7, induced by IL-6/STAT3 transactivation, promotes hepatocellular carcinoma aggressiveness through epithelial-mesenchymal transition. J Exp Clin Cancer Res. 2015;34:116.

24. Wu W, Chen F, Cui X, Yang L, Chen J, Zhao J, et al. LncRNA NKILA suppresses TGF-beta-induced epithelial-mesenchymal transition by blocking NF-kappaB signaling in breast cancer. Int J Cancer. 2018;143:2213-24.

25. Xia S, Wang C, Ni X, Ni Z, Dong Y, Zhan W. NONHSAT076754 aids ultrasonography in predicting lymph node metastasis and promotes migration and invasion of papillary thyroid cancer cells. Oncotarget. 2017;8: 2293-306.

26. Xiong J, Liu Y, Luo S, Jiang L, Zeng Y, Chen Z, et al. High expression of the long non-coding RNA HEIRCC promotes renal cell carcinoma metastasis by inducing epithelial-mesenchymal transition. Oncotarget. 2017:8:6555-63.

27. Zhuang J, Lu Q, Shen B, Huang X, Shen L, Zheng X, et al. TGFbeta1 secreted by cancer-associated fibroblasts induces epithelial-mesenchymal transition of bladder cancer cells through IncRNA-ZEB2NAT. Sci Rep. 2015;5:11924.

28. Huang W, Cui X, Chen J, Feng Y, Song E, Li J, et al. Long non-coding RNA NKILA inhibits migration and invasion of tongue squamous cell carcinoma cells via suppressing epithelial-mesenchymal transition. Oncotarget. 2016;7: 62520-32.

29. Lukanova A, Kaaks R. Endogenous hormones and ovarian cancer: epidemiology and current hypotheses. Cancer Epidemiol Biomarkers Prev. 2005;14:98-107.

30. Chao TK, Yo YT, Liao YP, Wang YC, Su PH, Huang TS, et al. LIM-homeobox transcription factor 1, alpha (LMX1A) inhibits tumourigenesis, epithelialmesenchymal transition and stem-like properties of epithelial ovarian cancer. Gynecol Oncol. 2013;128:475-82.

31. Tellez CS, Juri DE, Do K, Bernauer AM, Thomas CL, Damiani LA, et al. EMT and stem cell-like properties associated with miR-205 and miR-200 epigenetic silencing are early manifestations during carcinogen-induced transformation of human lung epithelial cells. Cancer Res. 2011;71:3087-97.

32. Yu J, Han Q, Cui Y. Decreased long non-coding RNA SPRY4-IT1 contributes to ovarian cancer cell metastasis partly via affecting epithelial-mesenchymal transition. Tumour Biol. 2017;39:1010428317709129.

33. Shi Y, Liu Y, Wang J, Jie D, Yun T, Li W, et al. Downregulated long noncoding RNA BANCR promotes the proliferation of colorectal Cancer cells via Downregualtion of p21 expression. PLoS One. 2015;10:e0122679.

34. Li L, Zhang L, Zhang Y, Zhou F. Increased expression of LncRNA BANCR is associated with clinical progression and poor prognosis in gastric cancer. Biomed Pharmacother. 2015;72:109-12.

35. Dhamija S, Diederichs S. From junk to master regulators of invasion: IncRNA functions in migration, EMT and metastasis. Int J Cancer. 2016;139:269-80.

36. Fujioka T, Takebayashi Y, Kihana T, Kusanagi Y, Hamada K, Ochi H, et al. Expression of E-cadherin and beta-catenin in primary and peritoneal metastatic ovarian carcinoma. Oncol Rep. 2001;8:249-55.

\section{Publisher's Note}

Springer Nature remains neutral with regard to jurisdictional claims in published maps and institutional affiliations.

Ready to submit your research? Choose BMC and benefit from:

- fast, convenient online submission

- thorough peer review by experienced researchers in your field

- rapid publication on acceptance

- support for research data, including large and complex data types

- gold Open Access which fosters wider collaboration and increased citations

- maximum visibility for your research: over $100 \mathrm{M}$ website views per year

At BMC, research is always in progress.

Learn more biomedcentral.com/submissions 